\title{
Studies on Genetic Diversity for Grain Yield and Nutritional Traits in Foxtail Millet Genetic Resources [Setaria italica (L.) Beauv.]
}

\author{
G.K. Pavan Kumar ${ }^{1 *}$, A.V.S. Durga Prasad ${ }^{1}$ and C.V. Chandra Mohan Reddy ${ }^{2}$ \\ ${ }^{1}$ Department of Genetics and Plant Breeding, Agricultural College, Mahanandi -518 502, \\ A.P., India \\ ${ }^{2}$ (Small millets), RARS, Nandyal - 518 501, A.P., India \\ *Corresponding author
}

\begin{abstract}
A B S T R A C T
Keywords

Foxtail millet,

Genetic divergence,

$\mathrm{D}^{2}$, Tocher's

method

Article Info

Accepted:

15 July 2019

Available Online:

10 August 2019

Hundred foxtail millet genetic resources were studied to assess the magnitude of genetic diversity for 18 metric traits using Mahalanobis $\mathrm{D}^{2}$ Statistic (Tocher's method). The results of the study showed that the genotypes were grouped into 11 distinct non-overlapping clusters suggesting availability of substantial genetic diversity. Maximum number of genetic resources were grouped in cluster I (43) followed by cluster II (37), cluster VI with six genetic resources, cluster IV, IX with four genetic resources each and the remaining were monogenotypic clusters III, V, VII, VIII, X and XI containing only single genetic resource indicating high degree of heterogeneity among the genotypes. Clusters $\mathrm{V}$ and XI showed maximum inter-cluster distance between them implying these genetic resources with high degree of genetic diversity may be utilized in inter-varietal hybridization programme. Considering the distribution of genotypes, it can be inferred that cross SiA 4044 x SiA 3222 alone has good potential of producing heterotic hybrids or transgressive segregants, depending on the gene action controlling the traits. The trait, number of grains / ear head followed by plant height contributed maximum towards total divergence indicating feasibility of improvement through those characters.
\end{abstract}

\section{Introduction}

Small millets are one of the ancient cereals grown by farmers mainly in the drought prone tracts of the globe. They can thrive in poor soils of arid and semi-arid regions, even in the hottest climates, where no other cereal can grow and yield high (Howarth et al., 2002). Nevertheless their cultivation is overlooked due to the impact of green revolution and is further stepped down to "Orphan cereals" (Brunda et al., 2015). But in recent times, due to heavy demand from health conscious consumers and their adaptability to climateresilient agriculture, there has been a rapid expansion of small millets area among the small millets, foxtail millet ranks second in terms of global production next to finger millet. The grains of this millet are an excellent source of quality proteins (leucine and methionine), beta carotene, minerals $(\mathrm{Ca}$, $\mathrm{Fe}, \mathrm{K}, \mathrm{Mg}$ and $\mathrm{Zn}$ ), antioxidants, dietary fibre, phytochemicals, vitamins (thiamine, riboflavin and niacin) and possess low glycemic index, a 
requisite for healthy human diet. The success of any plant breeding programme largely depends on the existence of diversity among the genotypes (Allard, 1960). The choice of parents is of paramount importance in breeding programme. Assessment of a large number of germplasm accessions for genetic diversity is of immense help in selection of diverse genotypes for hybridization programme. In order to identify diverse genetic resources, precise information on the nature and degree of genetic diversity is required which in turn helps the plant breeders in selecting the parents for targeted hybridization (Reddy et al., 2015).

The generalized distance concept of Mahalanobis' is based on multivariate analysis of quantitative traits. It is used to measure the genetic divergence and to classify the genetic stock into distinct groups. Intercrossing between more divergent parents is expected to generate a broad spectrum of variability and selection to be adopted in the segregating generations. Therefore the present study was undertaken to assess the genetic diversity in the utilized genetic resources of foxtail millet.

\section{Materials and Methods}

Field studies at Regional Agricultural Research Station, Nandyal, Andhra Pradesh utilizing hundred foxtail millet genetic resources were laid in a Augmented randomized complete block design (ARCBD) with four checks during Kharif, 2018 in order to study the genetic divergence by Mahalanobis' $\mathrm{D}^{2}$ statistics (Tocher's method) method. The experiment was carried out at an altitude of $211.3 \mathrm{~m}$ above mean sea level, latitude of $18.29^{\circ} \mathrm{N}$ and longitude of $78.29^{\circ} \mathrm{E}$ at RARS, Nandyal, A.P. The net plot size was $40 \times 3 \mathrm{~m}^{2}$ with a recommended spacing of $22.5 \mathrm{~cm} \times 10 \mathrm{~cm}$. The data was collected on five randomly selected plants per genetic resource for 18 metric traits viz., SCMR at 30
DAS, SCMR at 45 DAS, days to $50 \%$ flowering, plant height, panicle length, number of productive tillers /plant, days to maturity, number of grains / ear head, 1000 grain weight, protein, carbohydrate, calcium, magnesium, iron, zinc, copper, manganese and grain yield/ plant. Genetic divergence analysis was analysed through $\mathrm{D}^{2}$ statistic (Mahalanobis, 1936) and then genotypes were grouped on the basis of minimum generalized distance using Tochers' method (Rao, 1952). The data obtained was subjected to analysis using WINDOWSTAT 9.2 version software.

\section{Results and Discussion}

Hundred foxtail millet genetic resources, grouped into 11 distinct non-overlapping clusters using the Tocher's method (Table 1 and Fig. 2) suggested prevalence of substantial genetic diversity in the experimental material screened. Of the 11 clusters obtained, cluster I was the largest comprising of largest number of genetic resources (43) followed by clusters II with 37 genetic resources, cluster VI with six genetic resources, cluster IV, IX with four genetic resources each and the remaining were monogenotypic clusters III, V, VII, VIII, X and XI containing only single genetic resource each with no intra-cluster distances $\left(D^{2}\right.$ values) indicating high degree of heterogenity among the genetic resources. The formation of distinct monogenotypic clusters may be due to the fact that geographic barriers preventing gene flow or intensive natural and human selection for diverse and adaptable gene complexes must be responsible for this genetic diversity (Arunachalam and Ram, 1967).

The average intra and inter-cluster $\mathrm{D}^{2}$ values of 11 clusters were presented in Table 2 and Figure 1. The inter-cluster distances were higher than the intra-cluster distances indicating presence of wider genetic diversity between the clusters rather than with in the clusters. Intra-cluster $\mathrm{D}^{2}$ values ranged from 
0.00 (cluster III, V, VII, VIII, $\mathrm{X}$ and $\mathrm{XI}$ ) to 1256.89 (cluster IX). Maximum intra-cluster distance represented diversity among the genetic resources within cluster. Inter-cluster distance values varied from 705.65 (cluster VII and cluster X) to 69608.88 (cluster V and cluster XI). Hence the germplasm accessions between cluster V (SiA 4044) and cluster XI
( $\mathrm{SiA}$ 3222) possessing maximum inter cluster distance between them had high degree of genetic diversity and thus may be utilized under inter-varietal hybridization programme (transgressive breeding) for obtaining superior segregants. Similar results were reported by Mahanthesha et al., (2017b) and Amarnath et al., (2019).

Table.1 Clustering pattern of 100 foxtail millet [Setaria italica (L.) Beauv.] genotypes by Tocher's method

\begin{tabular}{|c|c|c|}
\hline $\begin{array}{c}\text { Cluster } \\
\text { No. }\end{array}$ & $\begin{array}{c}\text { No. of } \\
\text { genotypes }\end{array}$ & Genotype(s) \\
\hline $\mathbf{I}$ & 43 & $\begin{array}{l}\text { SiA 2713, SiA 4182, SiA 2663, SiA 3513, SiA 4068, SiA 3697, } \\
\text { SiA 3436, SiA 4009, SiA 4114, SiA 3965, SiA 3462, SiA 4141, } \\
\text { SiA 3827, SiA 3793, SiA 3753, SiA } 3156 \text { (C), SiA 3588, SiA } \\
\text { 2667, SiA 4036, SiA 4180, SiA 3749, SiA 3908, SiA 3560, SiA } \\
\text { 1266, SiA 3971, SiA 3894, SiA 3643, SiA 3674, SiA 3972, SiA } \\
\text { 3756, } \\
\text { SiA 3516, SiA 4045, SiA 4167, SiA 2864, SiA 3423, SiA 3465, } \\
\text { SiA 3420, SiA 3038, SiA 2844, Suryanandi (C), SiA 2849, SiA } \\
\text { 4027, SiA 4013. }\end{array}$ \\
\hline II & 37 & $\begin{array}{l}\text { SiA 2671, SiA 4020, SiA 2674, SiA 2757, SiA 3855, SiA 3639, } \\
\text { SiA 3559, SiA 3657, SiA 2745, SiA 3430, SiA 4063, SiA 3737, } \\
\text { SiA 2662, SiA 4179, SiA 3496, SiA 3498, SiA 3492, SiA 2737, } \\
\text { SiA 4155, SiA 2579, SiA 4181, SiA 3469, SiA 3851, SiA 3323, } \\
\text { SiA 3413, SiA 3754,SiA 805, SiA 2850, SiA 4107, SiA 3611, } \\
\text { SiA 3281, SiA 3701, SiA 3511, Prasad (C), SiA 4061, SiA 2697, } \\
\text { SiA 2856 }\end{array}$ \\
\hline III & 1 & SiA 3419 \\
\hline IV & 4 & SiA 3554, SiA 3085 (C), SiA 3969, SiA 3499 \\
\hline $\mathbf{V}$ & 1 & SiA 4044 \\
\hline VI & 6 & SiA 3580, SiA 3429, SiA 3627, SiA 3422, SiA 4016, SiA 2681 \\
\hline VII & 1 & SiA 3282 \\
\hline VIII & 1 & SiA 3409 \\
\hline IX & 4 & SiA 3577, SiA 3435, SiA 1244, SiA 4005 \\
\hline $\mathbf{X}$ & 1 & SiA 3291 \\
\hline XI & 1 & SiA 3222 \\
\hline
\end{tabular}


Table.2 Average intra and inter-cluster distances ( $\mathrm{D}^{2}$ values) among 11 clusters (obtained by Tocher's method) in 100 foxtail millet [Setaria italica (L.) Beauv.] genotypes

\begin{tabular}{|c|c|c|c|c|c|c|c|c|c|c|c|}
\hline $\begin{array}{c}\text { Cluster } \\
\text { No. }\end{array}$ & I & II & III & IV & $\mathbf{V}$ & VI & VII & VIII & IX & $\mathbf{X}$ & XI \\
\hline I & 575.46 & 65403.52 & 984.50 & 40149.59 & 1047.97 & 2539.69 & 67709.18 & 1370.74 & 19657.23 & 67509.34 & 68409.10 \\
\hline II & & 696.99 & 64751.46 & 3665.34 & 65847.75 & 46549.05 & 1316.85 & 68448.33 & 14637.99 & 2222.88 & 3287.49 \\
\hline III & & & 0.00 & 39675.33 & 1378.28 & 2667.98 & 68179.24 & 2369.24 & 19251.32 & 67773.11 & 69328.85 \\
\hline IV & & & & 566.06 & 40596.53 & 25849.76 & 4745.19 & 42863.47 & 4623.90 & 5456.28 & 6449.81 \\
\hline $\mathbf{V}$ & & & & & 0.00 & 2635.14 & 68922.66 & 1937.80 & 19989.14 & 68383.91 & 69608.88 \\
\hline VI & & & & & & 1139.38 & 48891.50 & 3694.50 & 10385.07 & 48832.64 & 50143.52 \\
\hline VIII & & & & & & & & 0.00 & 21798.52 & 68124.17 & 69031.28 \\
\hline IX & & & & & & & & & 1256.89 & 16562.42 & 17682.48 \\
\hline $\mathbf{X}$ & & & & & & & & & & 0.00 & 710.54 \\
\hline XI & & & & & & & & & & & 0.00 \\
\hline
\end{tabular}

Note: Diagonal values are intra-cluster distances. Off-diagonal values are inter-cluster distances 
Table.3 Mean values of eleven clusters estimated by Tocher's method in 100 foxtail millet [Setaria italica (L.) Beauv.] genotypes

\begin{tabular}{|c|c|c|c|c|c|c|c|c|c|c|c|c|c|c|c|c|c|c|}
\hline $\begin{array}{l}\text { Cluster } \\
\text { No. }\end{array}$ & $\begin{array}{l}\text { SCMR } \\
\text { at } 30 \\
\text { DAS }\end{array}$ & $\begin{array}{l}\text { SCMR } \\
\text { at } 45 \\
\text { DAS }\end{array}$ & $\begin{array}{c}\text { Days to } \\
50 \% \\
\text { lowering }\end{array}$ & $\begin{array}{c}\text { Plant } \\
\text { height } \\
(\mathbf{c m})\end{array}$ & $\begin{array}{c}\text { Panicle } \\
\text { length } \\
(\mathbf{c m})\end{array}$ & $\begin{array}{l}\text { Number o } \\
\text { productive } \\
\text { tillers } \\
\text { /plant }\end{array}$ & $\begin{array}{l}\text { Days to } \\
\text { maturity }\end{array}$ & $\begin{array}{l}\text { Number of } \\
\text { Grains / } \\
\text { ear head }\end{array}$ & $\begin{array}{c}1000 \\
\text { grain } \\
\text { weig } \\
\text { ht } \\
\text { (g) }\end{array}$ & $\begin{array}{l}\text { Protein } \\
\text { (g/ } \\
\text { 100g) }\end{array}$ & $\begin{array}{l}\text { Carbohyd } \\
\text { rate }(\mathrm{g} / \\
100 \mathrm{~g})\end{array}$ & $\begin{array}{c}\text { Calciun } \\
\text { (mg/ } \\
100 \mathrm{~g})\end{array}$ & $\begin{array}{l}\text { Magnesi } \\
\text { um (mg/ } \\
100 \mathrm{~g})\end{array}$ & \begin{tabular}{|c|} 
Iron \\
$(\mathrm{mg} / 100$ \\
$\mathrm{g})$
\end{tabular} & $\begin{array}{c}\text { Zinc } \\
\text { (mg/ } \\
\text { 100g) }\end{array}$ & $\begin{array}{c}\text { Copper } \\
(\mathrm{mg} / \\
100 \mathrm{~g})\end{array}$ & $\begin{array}{c}\text { Manga } \\
\text { nese } \\
(\mathrm{mg} / \\
\mathbf{1 0 0 g})\end{array}$ & $\begin{array}{c}\text { Grain } \\
\text { yield/ } \\
\text { plant } \\
\text { (g) }\end{array}$ \\
\hline I & 46.63 & 54.76 & 44.84 & 147.02 & 17.69 & 4.17 & 81.92 & 1559.98 & 2.95 & 14.22 & 63.63 & 24.98 & 14.91 & 9.41 & 3.31 & 1.15 & 2.31 & 10.52 \\
\hline II & 46.94 & 52.60 & 45.00 & 153.46 & 17.64 & 3.50 & 82.88 & 1814.37 & 2.96 & 14.08 & 61.27 & 22.92 & 15.51 & 11.97 & 3.13 & 1.36 & 2.34 & 13.09 \\
\hline III & 43.58 & 59.93 & 46.84 & 165.89 & 18.93 & 2.67 & 80.16 & 1561.54 & 2.79 & 14.86 & 50.29 & 16.21 & 9.71 & 5.93 & 2.58 & 0.88 & 2.03 & 8.79 \\
\hline IV & 44.15 & 56.35 & 45.38 & 153.28 & 19.16 & 4.58 & 83.12 & 1758.91 & 3.00 & 14.17 & 63.39 & 23.21 & 13.62 & 13.41 & 4.65 & 1.02 & 2.75 & 13.48 \\
\hline $\mathbf{V}$ & 40.83 & 51.01 & 49.84 & 151.36 & 19.20 & 5.33 & 85.16 & 1559.82 & 2.65 & 13.47 & 50.98 & 39.89 & 21.90 & 23.54 & 2.68 & 0.89 & 2.78 & 5.12 \\
\hline VI & 46.09 & 48.63 & 48.01 & 150.85 & 16.76 & 4.13 & 83.91 & 1600.67 & 2.96 & 13.83 & 59.38 & 24.62 & 14.40 & 15.92 & 3.31 & 1.83 & 2.26 & 10.23 \\
\hline VII & 44.73 & 50.66 & 36.09 & 128.11 & 13.53 & 1.48 & 81.91 & 1818.48 & 2.12 & 11.73 & 71.71 & 20.25 & 12.04 & 6.38 & 2.26 & 0.84 & 1.84 & 6.66 \\
\hline VIII & 43.63 & 46.41 & 49.84 & 123.16 & 11.40 & 1.97 & 70.16 & 1556.22 & 1.61 & 12.16 & 56.04 & 23.89 & 7.50 & 6.37 & 2.47 & 0.89 & 2.38 & 1.03 \\
\hline IX & 45.40 & 54.90 & 45.09 & 153.29 & 17.67 & 4.52 & 81.60 & 1697.03 & 2.91 & 14.26 & 57.29 & 22.00 & 20.41 & 9.54 & 3.70 & 1.44 & 2.47 & 12.44 \\
\hline $\mathbf{X}$ & 38.33 & 51.96 & 35.09 & 117.21 & 12.53 & 2.28 & 69.91 & 1816.48 & 2.13 & 16.54 & 52.63 & 20.25 & 9.64 & 6.78 & 2.58 & 0.84 & 2.30 & 6.30 \\
\hline XI & 46.63 & 65.91 & 32.09 & 109.96 & 14.53 & 1.04 & 60.16 & 1816.66 & 2.50 & 12.89 & 63.02 & 32.12 & 12.00 & 4.41 & 1.62 & 0.98 & 1.69 & 9.47 \\
\hline
\end{tabular}


Table.4 Contribution of different characters towards genetic divergence in 100 foxtail millet [Setaria italica (L.) Beauv.] genotypes

\begin{tabular}{|c|c|c|c|}
\hline S.NO. & Characters & No. of times ranked first & Contribution (\%) \\
\hline 1 & SCMR at 30 DAS & 71 & 1.43 \\
\hline 2 & SCMR at 45 DAS & 53 & 1.07 \\
\hline 3 & Days to $50 \%$ flowering & 18 & 0.36 \\
\hline 4 & Plant height & 661 & 13.35 \\
\hline 5 & Panicle length & 1 & 0.02 \\
\hline 6 & $\begin{array}{l}\text { Number of productive tillers } \\
\text { /plant }\end{array}$ & 0 & 0.01 \\
\hline 7 & Days to maturity & 35 & 0.71 \\
\hline 8 & Number of grains / ear head & 3210 & 64.85 \\
\hline 9 & 1000 grain weight & 0 & 0.01 \\
\hline 10 & Protein & 1 & 0.02 \\
\hline 11 & Carbohydrate & 403 & 8.14 \\
\hline 12 & Calcium & 177 & 3.58 \\
\hline 13 & Magnesium & 149 & 3.01 \\
\hline 14 & Iron & 157 & 3.17 \\
\hline 15 & Zinc & 0 & 0.01 \\
\hline 16 & Copper & 0 & 0.01 \\
\hline 17 & Manganese & 0 & 0.01 \\
\hline 18 & Grain yield/ plant & 14 & 0.28 \\
\hline
\end{tabular}


Fig.1 Intra and inter-cluster distance of 100 foxtail millet [Setaria italica (L.) Beauv.] genotypes in 11 clusters based on Tocher's method

\section{Tocher Method}

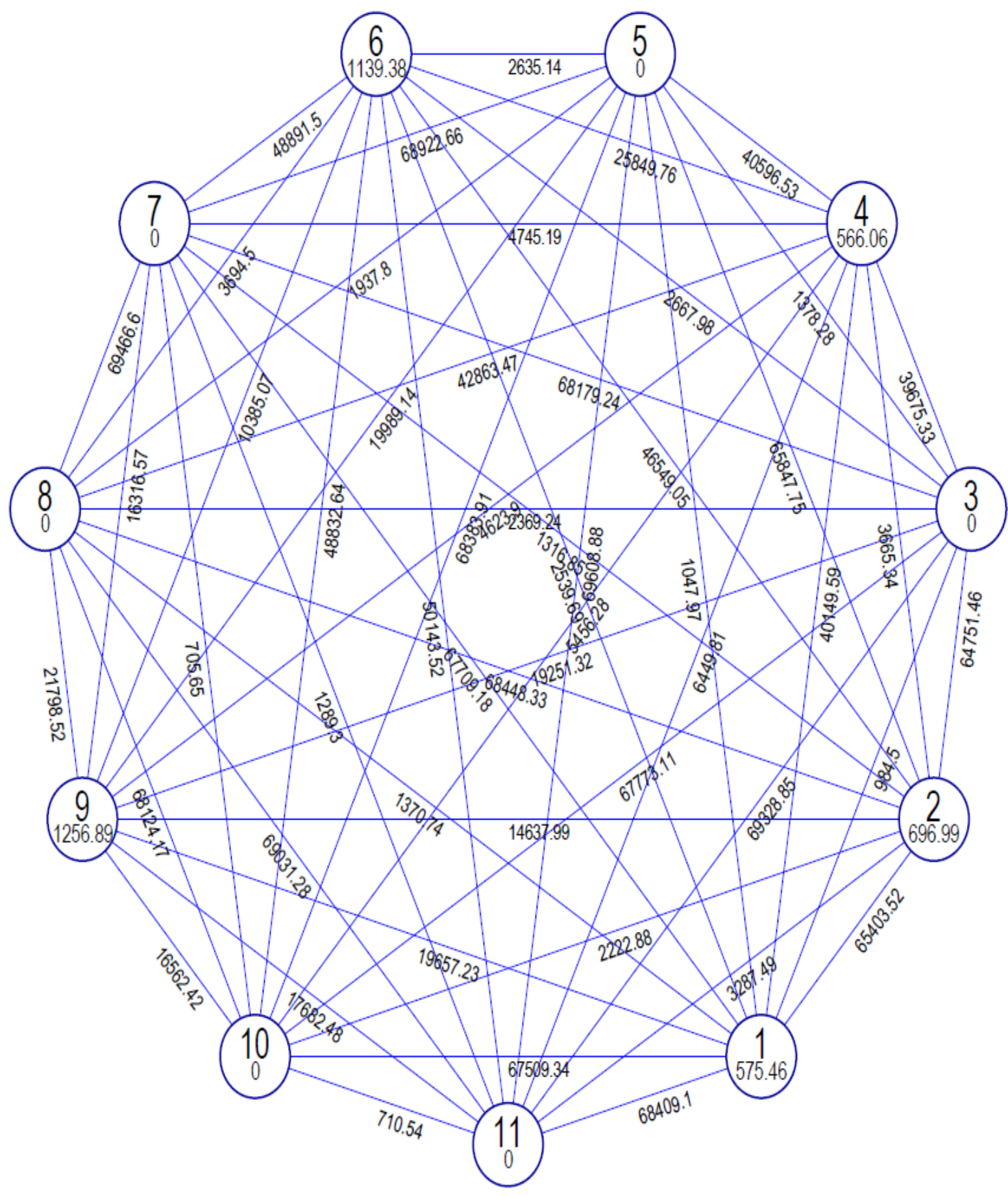

Mahalanobis Euclidean Distance (Not to the Scale) 
Fig.2 Dendrogram showing relationship among 100 foxtail millet [Setaria italica (L.) Beauv.] genotypes 11 clusters based on Mahalanobis' $\mathrm{D}^{2}$ values using Tocher's method

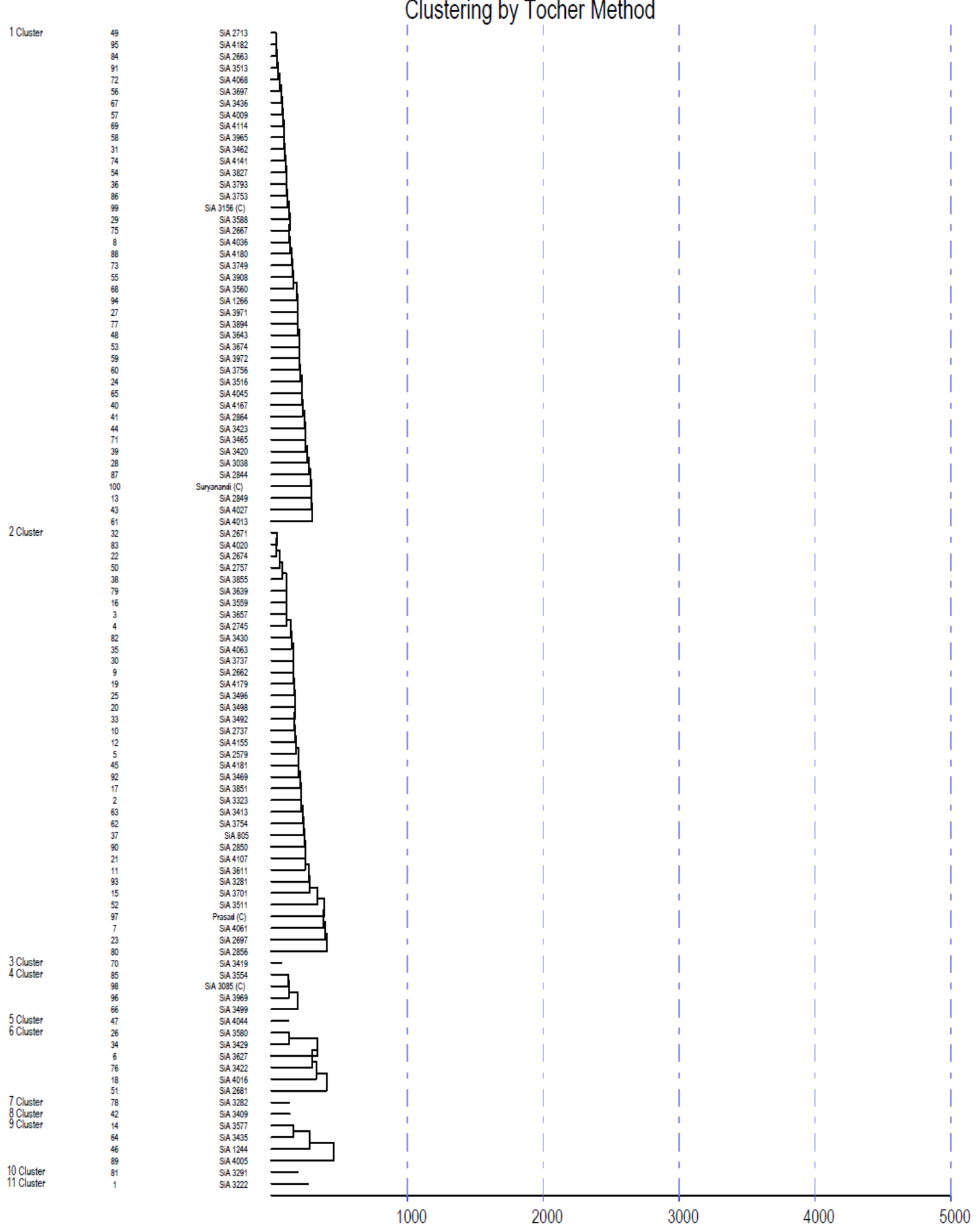


The cluster means values for 18 characters studied were presented in Table 3. Wide spectrum of variation was observed in clusters for the characters studied implying that the clusters formed were distinct. The cluster V reported maximum panicle length (19.2), productive tillers / plant (5.33) and highest contents of calcium (39.89), magnesium (21.9), iron (23.54) and manganese (2.78). Highest 1000 grain weight (3), zinc content (4.65) and grain yield / plant (13.48) were noticed in cluster IV. Cluster XI registered highest SCMR at 45 DAS (46.94) and desired earliness for days to $50 \%$ flowering (32.09) and maturity (60.16). Cluster VII registered highest number of grains / ear head (1818) and maximum carbohydrate content (71.71).

The clusters II, III, VI and X reported highest SCMR 30 DAS (46.94), plant height (165.9), copper content (1.83) and protein content (16.54) respectively. The result indicates that selection of genetic resources having desired values for particular trait could be made and utilized in the hybridization programme for improvement of that character. Therefore, hybridization between the selected germplasm accessions from divergent clusters is essential to judiciously combine all the targeted traits. Similar findings were reported by Kavya et al., (2017b) and Amarnath et al., (2019).

Based on Tocher's method, it can be inferred that the genetic resources between cluster $\mathrm{V}$ (SiA 4044) and cluster XI (SiA 3222) possessing maximum inter cluster distance between them had high degree of genetic diversity and thus may be utilized under intervarietal hybridization programme (transgressive breeding) for obtaining superior segregants (Table 4). In selecting genotypes for initiating any breeding programme, one should also consider the per se performance of those genotypes for yield and yield contributing characters along with genetic diversity.

\section{References}

Allard, R.W. 1960. Principles of Plant Breeding. John Wiley and Sons Inc., New York. pp. 485.

Amarnath, K., Prasad, A.V.S.D and Reddy, C.V.C.M. 2019. Assessment of genetic diversity in Indian Italian millet genetic resources [Setaria italica (L.) Beauv]. Electronic Journal of Plant Breeding, 10 (1): $83-91$.

Arunachalam, V and Ram, J. 1967. Geographical diversity in relation to genetic divergence in cultivated sorghum. Indian J. Genet. 27: 369-380.

Brunda, S.M., Kamatar, M.Y., Naveenkumar, K.L., Hundekar, R. 2015. Genetic diversity in the foxtail millet (Setaria italica) germplasm as determined by phonotypical traits. The Ecoscan. 8: 483-488.

Howarth, C.J and Yadav, R.S. 2002. Successful marker assisted selection for drought tolerance and diseases resistance in pearl millet. IGER Innovation: 18-21.

Kavya, P., Sujatha, M., Pandravada, S.R and Hymavathi, T.V. 2017. Exploiting genetic divergence in Italian millet [Setaria italica (L.) P. Beauv.] Elite germplasm lines: a neglected and a under utilised crop. Environment and Ecology. 35(3C): 2312-2315.

Mahanthesha, M., Sujatha, M., Pandravada, S.R and Meena, A.K. 2017. Study of genetic divergence in finger millet (Eleusine coracana (L.) Gaertn) germplasm. International Journal of Pure and Applied Bioscience. 5(3): 373377.

Marathee, J.P. 1993. Structure and characteristics of the world millet economy. Advances in small millets (Riley KW, Gupta SC, Seetharam A and Mushonga JN, eds.). New Delhi, India: Oxford \& IBH. pp. 159-178. 
Rao, C.R. 1952. Advanced Statistical Methods in Biometrical Research. John Willey and Sons, Inc., New York. pp. 357-363.

Reddy, C.V.C.M., Pullibai, P., Manjunath, J and Munirathnam, P. 2015. Genetic diversity and genotype by trait analysis for yield and yield attributing traits in Italian millet (Setaria italica (L.) Beauv). International Journal of Agricultural Innovations and Research. 3(6): 1726-1730.

\section{How to cite this article:}

Pavan Kumar, G.K., A.V.S. Durga Prasad and Chandra Mohan Reddy, C.V. 2019. Studies on Genetic Diversity for Grain Yield and Nutritional Traits in Foxtail Millet Genetic Resources [Setaria italica (L.) Beauv.]. Int.J.Curr.Microbiol.App.Sci. 8(08): 1990-1909.

doi: https://doi.org/10.20546/ijcmas.2019.808.223 